Review Article

\title{
Skeletal Muscle and Lymphocyte Mitochondrial Dysfunctions in Septic Shock Trigger ICU-Acquired Weakness and Sepsis-Induced Immunoparalysis
}

\author{
Quentin Maestraggi, ${ }^{1,2}$ Benjamin Lebas, ${ }^{2,3}$ Raphaël Clere-Jehl,, 1,2 \\ Pierre-Olivier Ludes, ${ }^{2,3}$ Thiên-Nga Chamaraux-Tran, ${ }^{2,3,4}$ Francis Schneider, ${ }^{1,2}$ \\ Pierre Diemunsch, ${ }^{2,3}$ Bernard Geny, ${ }^{2,5}$ and Julien Pottecher ${ }^{2,3}$ \\ ${ }^{1}$ Hôpitaux Universitaires de Strasbourg, Hôpital de Hautepierre, Service de Réanimation Médicale, avenue Molière, \\ 67098 Strasbourg Cedex, France \\ ${ }^{2}$ Université de Strasbourg, Fédération de Médecine Translationnelle de Strasbourg (FMTS), Faculté de Médecine, \\ Institut de Physiologie, Equipe d'Accueil 3072 "Mitochondrie, Stress Oxydant et Protection Musculaire", 11 rue Human, \\ 67000 Strasbourg, France \\ ${ }^{3}$ Hôpitaux Universitaires de Strasbourg, Hôpital de Hautepierre, Service d'Anesthésie-Réanimation Chirurgicale, avenue Molière, \\ 67098 Strasbourg Cedex, France \\ ${ }^{4}$ Institut de Génétique et de Biologie Moléculaire et Cellulaire (IGBMC), CNRS UMR7104, INSERM U964, Université de Strasbourg, \\ Illkirch, France \\ ${ }^{5}$ Hôpitaux Universitaires de Strasbourg, Nouvel Hôpital Civil, Service de Physiologie et d'Explorations Fonctionnelles, \\ 1 Place de l'Hôpital, 67091 Strasbourg Cedex, France \\ Correspondence should be addressed to Julien Pottecher; julien.pottecher@chru-strasbourg.fr
}

Received 1 February 2017; Revised 16 March 2017; Accepted 23 April 2017; Published 15 May 2017

Academic Editor: Thomas Griffith

Copyright ( 2017 Quentin Maestraggi et al. This is an open access article distributed under the Creative Commons Attribution License, which permits unrestricted use, distribution, and reproduction in any medium, provided the original work is properly cited.

Fundamental events driving the pathological processes of septic shock-induced multiorgan failure (MOF) at the cellular and subcellular levels remain debated. Emerging data implicate mitochondrial dysfunction as a critical factor in the pathogenesis of sepsis-associated MOF. If macrocirculatory and microcirculatory dysfunctions undoubtedly participate in organ dysfunction at the early stage of septic shock, an intrinsic bioenergetic failure, sometimes called "cytopathic hypoxia," perpetuates cellular dysfunction. Short-term failure of vital organs immediately threatens patient survival but long-term recovery is also severely hindered by persistent dysfunction of organs traditionally described as nonvital, such as skeletal muscle and peripheral blood mononuclear cells (PBMCs). In this review, we will stress how and why a persistent mitochondrial dysfunction in skeletal muscles and PBMC could impair survival in patients who overcome the first acute phase of their septic episode. First, muscle wasting protracts weaning from mechanical ventilation, increases the risk of mechanical ventilator-associated pneumonia, and creates a state of ICU-acquired muscle weakness, compelling the patient to bed. Second, failure of the immune system ("immunoparalysis") translates into its inability to clear infectious foci and predisposes the patient to recurrent nosocomial infections. We will finally emphasize how mitochondrial-targeted therapies could represent a realistic strategy to promote long-term recovery after sepsis.

\section{Introduction}

Sepsis is a potentially lethal condition defined by lifethreatening organ dysfunction caused by a dysregulated host response to infection [1]. The causal microorganism can either be bacterial, fungal, viral, or parasitical. Septic shock is a particularly severe form of sepsis associated with higher mortality rates. The classical definition described septic shock as the cardiovascular dysfunction associated with infection, if another cause of shock was ruled out $[2,3]$. Severe sepsis 
referred to sepsis complicated by organ dysfunction and was an intermediary form of the disease whose severity lied between sepsis and septic shock. According to the recent Third International Consensus Definitions for Sepsis and Septic Shock (Sepsis-3), septic shock can be defined using the clinical criteria of arterial hypotension (requiring vasopressor to maintain mean arterial blood pressure over $65 \mathrm{mmHg}$ ) associated with a serum lactate level greater than $2 \mathrm{mmol} / \mathrm{L}$ despite adequate fluid resuscitation [1, 4]. Under Sepsis-3 terminology, sepsis is clinically characterized by a suspected or documented infection and an acute increase of $\geq 2$ SepsisRelated Organ Failure Assessment [5] (SOFA) points (a proxy for organ dysfunction). The term "severe sepsis" now becomes superfluous and its use is no longer recommended. For the sake of consistency, sepsis, severe sepsis, and septic shock terminologies are used in the following review according to the publication date of the reference cited (either before or after 2016).

The combination of hypotension, vasopressor use, and lactate level greater than $2 \mathrm{mmol} / \mathrm{L}$, defining septic shock under Sepsis-3 terminology, is associated with hospital mortality rates consistently higher than $40 \%$. These prohibitive mortality rates remain roughly unchanged despite extensive fundamental research and on-going medical progress. Understanding the pathophysiology of septic shock-induced multiorgan failure (MOF) thus remains a prerequisite to improve its outcome.

However, fundamental events driving the pathological processes of septic shock-induced MOF at the cellular and subcellular levels remain controversial [6,7]. Indeed, autopsy studies of patients dying in the intensive care unit (ICU) from septic shock-induced MOF reveal only marginal histological injury [8]. If macrocirculatory [9] and microcirculatory [10] dysfunctions undoubtedly participate in organ dysfunction at the early stage of septic shock, an intrinsic bioenergetic failure (termed cytopathic hypoxia [11]) perpetuates cellular dysfunctions [12]. Thereby, cells become unable to consume available oxygen due to mitochondrial shutdown [13], consisting of impaired electron transport chain [14] (ETC), sustained reactive oxygen species (ROS) generation [15], and increased apoptosis [16].

Sepsis-induced failure of vital organs (heart, kidney, and liver) threatens immediately patient's survival. However midand long-term recovery is also hindered by persistent dysfunction of crucial organs, traditionally considered as nonvital, such as skeletal muscle or peripheral blood mononuclear cells (PBMCs). Concomitant dysfunctions in skeletal muscles (either locomotive or respiratory) and PBMCs fuel a feedforward paresis-infection cycle, which ultimately leads to death.

However, comprehensively addressing septic shockinduced skeletal muscle and PBMC dysfunctions remains an unmet need. Therefore, our goals are to focus on these specific points and to stress how and why cellular and subcellular failure impair ICU outcome in patients who overcome the acute phase of septic shock. The role of persistent mitochondrial dysfunction in skeletal muscles and PBMC is specifically scrutinized. To conclude, we emphasize how mitochondrialtargeted therapies could improve long-term recovery after septic shock.

\section{Mitochondrial Homeostasis in Health}

Mitochondria are ubiquitous powerhouses of eukaryotic cells and they provide organisms with adenosine triphosphate (ATP) by using a group of enzymes, gathered in the mitochondrial electron transport chain (ETC), which transform transmembrane electron potential $\left(\Delta \Psi_{\mathrm{m}}\right)$ in biochemical energy. Besides baseline oxidative phosphorylation, spare respiratory capacity (SRC) represents the mitochondrial phosphorylation reserve, available to cope with an extra metabolic demand.

A small part of oxygen, not used for oxidative phosphorylation, leads to the mitochondrial production of ROS at complexes I and III through uncoupling [17]. Depending on the amount and duration of ROS production, oxidative stress may either play a protective or deleterious role on mitochondrial signalling [18-20]. Intramitochondrial level of ROS is tightly regulated by several antioxidant enzymes like manganese superoxide dismutase $\left(\mathrm{SOD}_{2}\right)$ or glutathione oxidase [21].

Mitochondrial hormesis (otherwise named mitochondrial biogenesis) is a cellular programed process that adjusts energy production by synthesis of new organelles and regulation of interorganelle interactions. The mitochondrial response to stressors (including sepsis) is sharply orchestrated by peroxisome proliferator-activated receptor- $\gamma$ coactivator $1 \alpha$ (PGC- $1 \alpha)$. PGC- $1 \alpha$ is a transcriptional coregulator, which both modulates nuclear-encoded mitochondrial enzymes and controls mitochondrial desoxyribonucleic acid (mtDNA) transcription after interacting with mitochondrial transcription factor A (Tfam) [22]. PGC- $1 \alpha$ expression is finely tuned by the energetic balance of the cell [23]. Furthermore mitochondrial biogenesis depends on intact immune system such as functionally active Toll-like receptors (TLRs) since genetically engineered TLR- 4 deficient mice cannot activate mitochondrial recovery [24].

Other functions of mitochondria include synthesis of steroid hormones [25], intracellular calcium homeostasis, and induction of cell death through both the apoptotic (release of cytochrome c [12]) and the necrotic (massive mPTP opening [26]) pathways.

\section{Sepsis-Induced Mitochondrial Dysfunction and Organ Failure}

Mitochondrial dysfunction encompasses multiple aspects of disturbed cellular homeostasis, including decreased ATP production, increased generation of ROS, calcium dysregulation, and mtDNA damage [28]. Considering that mitochondria are the main site of whole body oxygen consumption [22], their role has been evoked to explain oxygen misuse and "cytopathic hypoxia" during sepsis [12].

\subsection{A Coordinated Succession of Events}

3.1.1. Initial Multiorgan Failure. In vitro, sepsis-associated systemic inflammation produces ROS and reactive nitrogen species (RNS) primarily within the cytosolic NADPH oxidase 


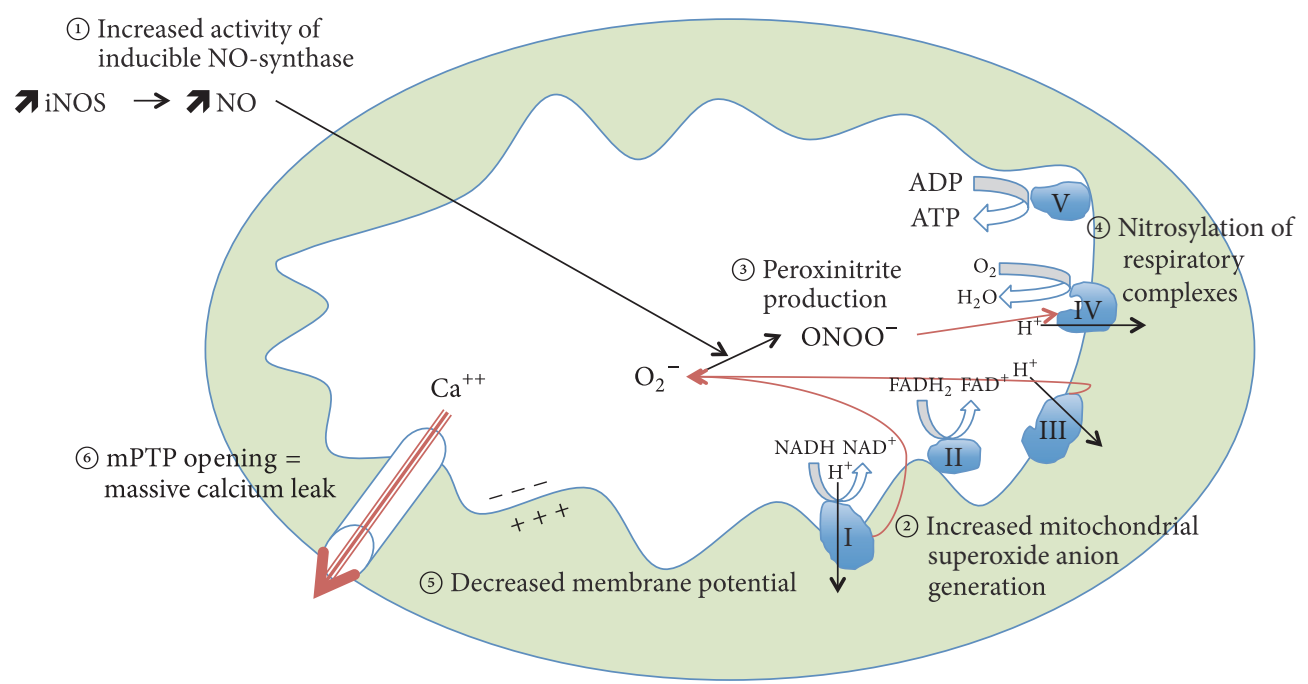

FIGURE 1: Succession of events leading to sepsis-induced mitochondrial dysfunction. (1) Increased activity of inducible NO-synthase. (2) Increased mitochondrial superoxide anion generation. (3) Production of peroxynitrite. (4) Nitrosylation of respiratory chain complexes. (5) Decreased membrane potential. (6) Opening of mitochondrial transition pore. Greek numbers refer to mitochondrial complexes. iNOS: inducible nitric oxide synthase; NO: nitric oxide; $\mathrm{O}_{2}{ }^{-}$: superoxide anion; $\mathrm{ONOO}^{-}$: peroxynitrite; $\mathrm{O}_{2}$ : oxygen; ADP: adenosine diphosphate; ATP: adenosine triphosphate; $\mathrm{H}^{+}$: proton; $\mathrm{FADH}_{2}$ : reduced flavin-adenine dinucleotide; $\mathrm{FAD}^{+}$: oxidized flavin-adenine dinucleotide; $\mathrm{NADH}$ : reduced nicotinamide adenine dinucleotide; $\mathrm{NAD}^{+}$: oxidized nicotinamide adenine dinucleotide; $\mathrm{Ca}^{++}$: ionized calcium; $\mathrm{mPTP}$ : mitochondrial permeability transition pore.

[29] but also in mitochondria through disruption of the ETC [30]. Both ROS and RNS trigger sepsis-induced strand damage of mitochondrial DNA and induce nitrosylation [31] of mitochondrial ETC proteins.

Due to its vicinity of the ETC and its lack of protection by histones, mitochondrial DNA is very susceptible to oxidative and nitrosative stress [32, 33]. Damaged mtDNA translates into defective mitochondrial respiratory complexes, perpetuating cellular bioenergetic crisis and increased ROS production. Moreover, fragmented mtDNA can escape the mitochondrial matrix through intermittent opening of the $\mathrm{mPTP}$ and induce both intracytosolic and systemic signalling [33]. Indeed, mtDNA acts as a Danger-Associated Molecular Pattern (DAMP) in animal models of sepsis [34] and triggers both intracellular inflammasome activation (through NODlike receptor family, pyrin domain containing 3, NLRP3 [35]) and remote organ injury (through Toll-like receptor 9 (TLR9) binding [36]).

In vitro [37], in animal models [38], prolonged inflammation induces decreased mitochondrial activity through increased nitric oxide (NO) levels and superoxide anion release, which combine to form the highly reactive peroxynitrite. Decreased mitochondrial activity is also worsened by inflammation-triggered ROS-mediated reduced gene transcription of mitochondrial-targeted genes. Indeed, dysfunctional ETC, reduced ATP levels, and reduced number of mtDNA copies following LPS exposure in mice can be abrogated with either overexpression of mitochondrial superoxide dismutase, iNOS inhibition, or peroxynitrite scavenger [39].

In humans, it is hypothesized that dysfunctional mitochondrial respiratory chain results in reduced ATP turnover and metabolic shutdown. When metabolic shutdown is sufficiently intense to prevent adequate production of ATP, cells can no longer maintain energy availability for essential cellular functions and go into either necrotic or apoptotic cell death pathways [40], triggering organ failure and death (Figure 1).

However, the initial multiorgan metabolic shutdown may be revocable, since most organs recover when the septic patient overcomes his infectious episode [41]. At the subcellular level, decreased activity of the mitochondrial ETC in human sepsis may be reversible as well. Indeed, endothelial cells perfused in vitro with serum from septic shock patients show a $40 \%$ decrease in mitochondrial respiration, which is restored with both a NOS inhibitor and a blocker of the poly(ADP-ribose) synthase pathway [42]. Conversely, the nitrosylation of mitochondrial ETC complexes might become persistent when the mitochondrial glutathione pool is oxidized [31, 43]. Even if human data are scarce [43] many experimental findings converge to persistent S-nitrosylation of complex I as a trigger for persistent ETC dysfunction, increased superoxide production [44], energy failure crisis, mPTP opening, and release of proapoptotic factors $[43,45]$.

3.1.2. Subsequent Activation of Mitochondrial Hormesis. If the organism (either animal or human) survives, as septic shock recovers, prolonged exposure to lower levels of NO activates mitochondrial biogenesis that promotes mitochondrial proliferation and a progressive increase in cell metabolism [46] (Figure 2). Consecutive to the activation of the mitochondrial biogenesis program, expression of NRF-1, NRF- 2, PGC-1 $\alpha$, and Tfam accompanies restoration of mtDNA copy number [22]. This has elegantly been demonstrated by Bartz et al. in a murine model of Staphylococcus aureus sepsis [47]. 


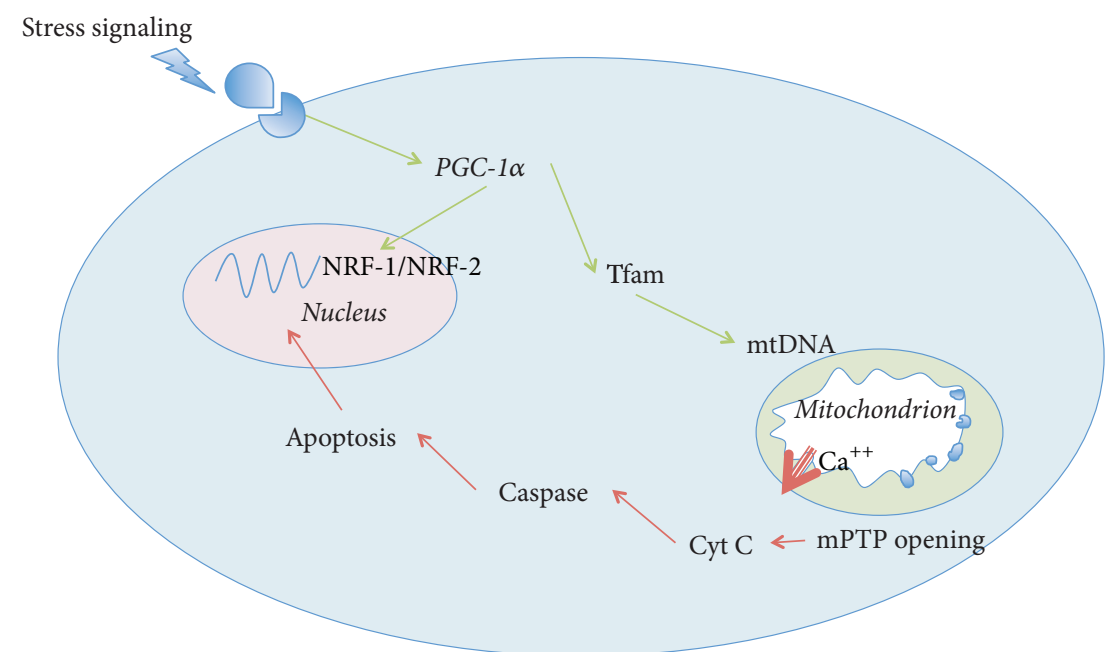

FIGURE 2: Nucleus-mitochondria crosstalk for apoptosis and mitochondrial hormesis induction: key role of PGC-1 $\alpha$, Tfam, and NRF-1/2. (i) Activation of caspase-3 and caspase-7 after mPTP opening leading to apoptosis. (ii) Activation of mitochondrial hormesis in response to stress stimulation (i.e., $\beta$-adrenergic). PGC- $1 \alpha$ plays a pivotal role for transcription of both nuclear and mitochondrial genes leading to increased mitohormesis. PGC- $1 \alpha$ : peroxisome proliferator-activated receptor- $\gamma$ coactivator $1 \alpha$; NRF-1/2: nuclear respiratory factor $1 / 2$; Tfam: mitochondrial transcription factor A; mtDNA: mitochondrial desoxyribonucleic acid; $\mathrm{Ca}^{++}$: ionized calcium; mPTP: mitochondrial permeability transition pore; Cyt c: cytochrome c.

The switch from mitochondrial metabolic shutdown to mitochondrial biogenesis is finely regulated and orchestrated by many factors that ultimately depend on sepsis severity and resilience of the organism [48] such as age, comorbidities, and genetic factors (e.g., mitochondrial haplotype in man [49]). The resulting signals will determine mitochondrial mass, number, size, distribution, and phenotype across cell types including skeletal muscle cells [50] and PBMCs [16] in ICU patients. Some mitochondrial haplotypes seem to convey an evolutionary benefit. For instance, in Newcastle upon Tyne (UK) haplogroup $\mathrm{H}$ was a strong independent predictor of better outcome during severe sepsis in 150 consecutive patients, conferring a 2.12 -fold (95\% CI 1.02-4.43) increased chance of survival [49].

3.2. Sepsis-Induced, Mitochondrial-Driven ICU-Acquired Muscle Weakness in Critically Ill Patients. ICU-acquired weakness is now a recognized clinical consequence of skeletal muscle mitochondrial dysfunction [51], which occurs simultaneously in respiratory (diaphragm, intercostal) and locomotive (vastus lateralis) muscles. ICU-acquired weakness undoubtedly contributes to worse mid- and longterm prognosis in patients with septic shock [52]. As recently underlined by Batt et al., ICU-acquired weakness has become a priority research focus in critical care [53]. For instance, ICU-acquired, sepsis-induced diaphragmatic dysfunction was strongly associated with increased ICU and hospital mortality [54]. Moreover, ICU-acquired limb weakness is an important patient-centered outcome with clear implications for quality of life [52]. In a prospective multicentre study, Ali et al. established that ICU-acquired weakness was independently associated with hospital mortality (odds ratio [OR], 7.8; 95\% confidence interval [CI], 2.4-25.3; $P<0.001$ ) even after adjustment for severity of illness [55]. Handgrip dynamometry providing a concise measure of global strength was independently associated with hospital mortality (OR, 4.5; 95\% CI, 1.5-13.6; $P<0.007)$. Muscular weakness and functional disability can persist as long as five years after critical illness [56].

\subsubsection{Sepsis-Induced, Mitochondrial-Driven ICU-Acquired} Diaphragm Weakness. As it extends ventilator-dependent duration, ICU-acquired diaphragm weakness may increase persistent functional limitation, the incidence of ventilatorassociated pneumonia, and healthcare-associated morbidity.

In a murine model of septic shock, Zolfaghari et al. demonstrated that maximal force generation was reduced and fatigue accelerated in ex vivo diaphragm muscle strips from septic mice, together with lower mitochondrial $\Delta \psi \mathrm{m}$ and phosphorylation capacity [57]. Sepsis-induced respiratory muscle proteolysis (mediated by calpain, caspase3 , and ubiquitin-proteasome system) may be exacerbated by mechanical ventilation as an additional mechanism for acquired diaphragm weakness [58] in both animal models [59] and clinical situations [60].

Even if clinical evidence of sepsis-induced direct diaphragm mitochondrial dysfunction is still lacking in man, mechanical ventilation was shown to induce profound defects in diaphragm mitochondrial biogenesis and cytochrome $c$ oxidase content in ventilated brain-dead organ donors [60]. Thus, sepsis and mechanical ventilation may have synergistic deleterious effects on diaphragm mitochondrial function in ventilated septic shock patients.

\subsubsection{Sepsis-Induced, Mitochondrial-Driven ICU-Acquired} Limb Weakness. Concerning limb muscles, deficits in both strength and size may exceed those seen in the diaphragm. For instance, Mofarrahi et al. reported reduced resistance 
to increased $\mathrm{Ca}^{2+}$ load and deeper biogenesis reduction in locomotor muscles (soleus and tibialis anterior) compared to diaphragm after a LPS challenge in mice [61]. Similar findings were reported among patients with sepsis [62].

Experimental findings suggest that prolonged and unopposed inflammatory processes during sepsis amplify TNF $\alpha$ production, whose catabolic effects on murine skeletal muscle trigger both decreased mitochondrial respiration and impaired biogenesis (through PGC- $1 \alpha$ inactivation [61]). As such, sustained exposure of murine skeletal myotubes to $\mathrm{TNF} \alpha$ induces reduced expression of oxidative phosphorylation subunits [63] in a NFא-B-dependent manner. Some evidence also suggests that a circulating factor (either $\mathrm{NO}$ or/and $\mathrm{IL}_{6}$ and/or mtDNA) may be at work to explain widespread skeletal muscle protein loss (myosin breakdown, proteolysis) [64] and altered mitochondrial function [16, 43] in septic shock patients.

However, based on recent data from our team and others, implication of a circulating plasmatic factor remains unclear to account for muscular mitochondrial ETC decreasing activity in both experimental models and septic shock patients. For instance, in a short-term CLP-induced murine septic shock model, ex vivo mitochondrial assessments showed neither reduced enzymatic activities, altered mitochondrial respiration, nor reduced tolerance to calcium loading [65]. Moreover, exposure of rodents skeletal muscle mitochondria with plasma of septic shock patients had no significant effect on muscle mitochondrial respiration [66].

Pioneering studies from Mervyn Singer's laboratory established that mitochondrial ETC was severely impaired in skeletal muscle biopsies from septic shock patients [43], harvested within $24 \mathrm{~h}$ of ICU admission. Furthermore, mitochondrial complex I activity had a significant inverse correlation with both shock severity and $\mathrm{NO}$ production and had a significant positive correlation with concentrations of intracellular antioxidant concentration and ATP content. Similarly, in a recent human quadriceps comparative study, Jiroutková et al. confirmed the dysfunction in mitochondrial respiration, showing functional impairments and quantitative defects of respiratory complexes in muscle of septic patients compared to controls [67]. It must be stressed that in both Brealey et al.s[43] and Jiroutková et al.s [67] clinical studies, muscle samples were collected more than $24 \mathrm{~h}$ after sepsis onset, differing in this way from many experimental "acute" models (including ours [65]) and potentially explaining contrasting results [68].

Using microarray analysis the Singer's team consequently demonstrated that septic shock survivors were able to activate mitochondrial hormesis pathways (PGC- $1 \alpha$ and $\mathrm{SOD}_{2}$ ) that counteract early decrease in mitochondrial ETC [50]. On the opposite, sepsis-survivors with protracted critical illness and ICU-acquired weakness still demonstrate a 50\% reduction of oxidative phosphorylation in their vastus lateralis muscles [67]. Pharmacological induction of mitochondrial hormesis might therefore be an interesting way to overcome both the acute septic phase and the subsequent long-lasting ICUacquired weakness and therefore stands for a promising research to promote muscle recovery in critical illness.
3.3. Sepsis-Induced, Mitochondrial-Driven Immunosuppression. As mitochondria used to be ancient endosymbiotes, defence mechanisms from the host immune response directed towards the non-self also recognize molecular patterns shared by mitochondria (containing many DAMPs) and pathogen agents (PAMPs) [69]. The immune system and its interactions with extracellular mitochondrial components (mtDNA [70], formyl peptide [36], and Tfam [71]) thus play a crucial role in inducing both initial widespread inflammatory response (which contributes to mitochondrial dysfunction through oxidative stress) and later mitochondrial recovery and hormesis.

Initially, exhaustion of the immune system leads to immunoparalysis. Potential mechanisms of immune suppression in patients with septic shock include shift from proinflammatory to anti-inflammatory phenotype, lymphocyte mitochondrial energy and apoptosis-induced depletion of CD4 positive cells and dendritic cells [8]. For instance, in spleens removed after the death of septic shock patients, Hotchkiss et al. observed that the duration of the septic episode was significantly associated with the depletion of B cells and CD4 T cells [72].

\subsubsection{Sepsis-Induced, Mitochondrial-Driven Lymphocyte} Apoptosis. Septic shock is classically associated with lymphopenia [73], which appears at the early stage of the disease [74] and contributes to the Compensatory AntiInflammatory Response Syndrome (CARS). CARS is now considered to be developing in parallel with the proinflammatory response, as early as sepsis onset. This "compensatory" anti-inflammatory response leads to a deep, sepsis-induced immunosuppression, which may be responsible for late deaths [75]. Lymphopenia is part of CARS and results from apoptosis of roughly all classes of lymphocytes, especially $\mathrm{B}$ and $\mathrm{CD}^{+} \mathrm{T}$ cells [72]. The triggers of this apoptosis seem to be multiple, involving death receptor as well as mitochondrial-mediated apoptosis.

In a murine sepsis model of caecal ligation and puncture, Chang et al. have shown that downregulation of genes involved in mitochondrial-mediated apoptosis (Bim-/mice) provided a decrease in lymphocytes apoptosis and a better mice survival [76]. Mitochondrial-mediated apoptosis has also been shown to be enhanced by the rate of circulating histones, which induce mitochondrial injury in vitro [77].

In humans, involvement of mitochondrial-mediated apoptosis in sepsis has been evidenced by a caspase-9mediated profound progressive loss of $\mathrm{B}$ and $\mathrm{CD} 4+\mathrm{T}$ cells [72]. In 16 severe sepsis patients (13 of whom matching the updated septic shock definition), lymphocyte apoptosis resulted from both a downregulation of genes inhibiting mitochondrial-mediated apoptosis $(B c l-2$ and $B c l-x l)$ and an upregulation of proapoptotic genes (Bim, Bid, and Bak) [78]. Moreover, pharmaceutically decreasing $B a k / B c l-2$ and $\mathrm{Bax} / \mathrm{Bcl}$-xl ratios could prevent apoptosis PBMC in septic shock patients [79].

3.3.2. Sepsis-Induced, Mitochondrial-Driven Impaired Lymphocyte Function. Lymphocyte mitochondrial dysfunction has been shown in sepsis and was ascertained by an early 


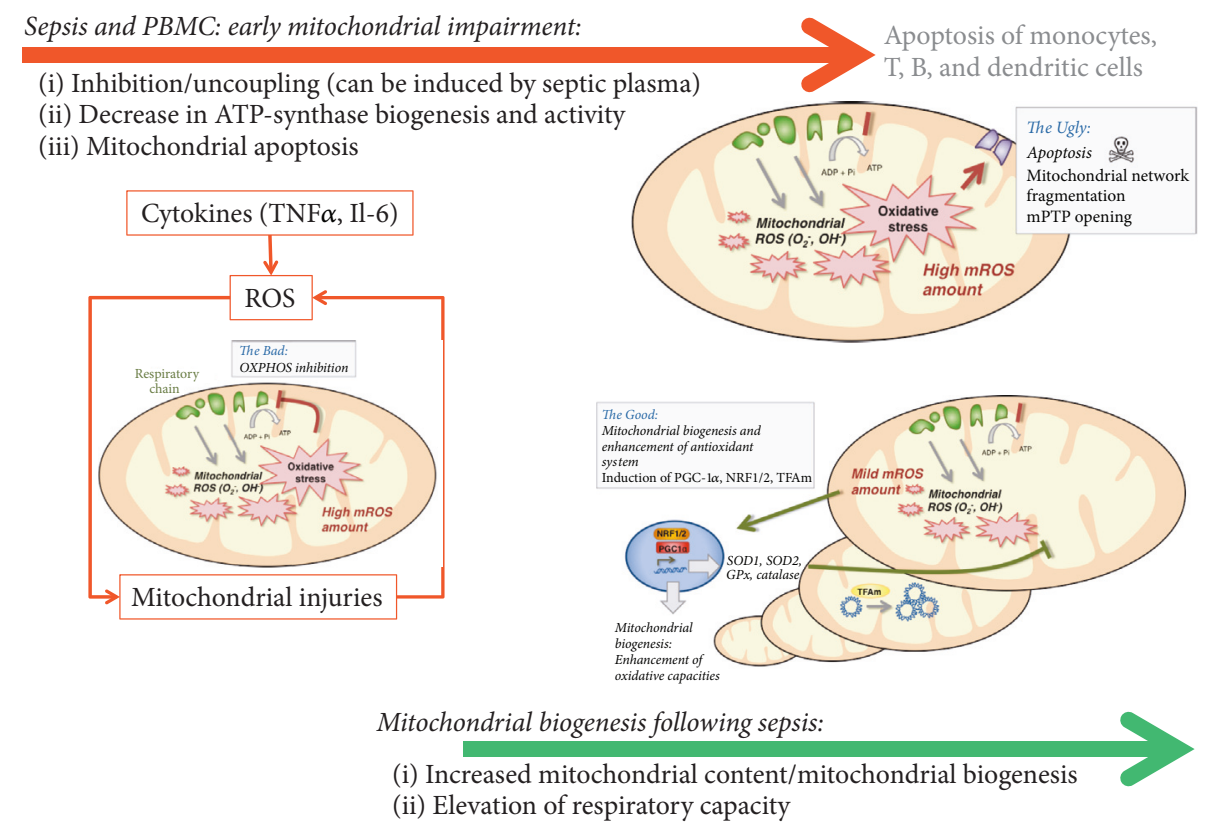

FIGURE 3: Time course of mitochondrial impairment and mitochondrial biogenesis following sepsis in peripheral blood mononuclear cells (PBMCs). TNF $\alpha$ : tumor necrosis factor $\alpha$; Il-6: interleukin-6; ROS: reactive oxygen species; SOD: superoxide dismutase, GPx: glutathione peroxidase; NRF-1/2: nuclear respiratory factor $1 / 2$; PGC- $1 \alpha$ : peroxisome proliferator-activated receptor- $\gamma$ coactivator $1 \alpha$; Tfam: mitochondrial transcription factor A. Adapted from Lejay et al. with permission [27].

decrease in mitochondrial respiratory capacity. Garrabou et al. observed a $16 \%$ decrease in spontaneous cell oxygen consumption in PBMCs from severe sepsis patients. Concurrent apoptosis process was shown in septic patients by an elevated caspase-3 activity and by a much higher percentage of cells with depolarized mitochondria. No change in cell mitochondrial content was observed at that stage [16]. Japiassú et al. pointed out a $43 \%$ decrease in cell respiration, specifically associated with reduced ATP synthesis in immune cells of septic shock patients compared with controls. This reduced energy synthesis came along with a $49 \%$ reduction in $\mathrm{F}_{1} \mathrm{~F}_{0}$ ATP-synthase activity in septic patients [80]. The same study found that reduction in ATP synthesis was significantly associated with organ failure and death, so that the degree of mitochondrial impairment was correlated with the clinical outcome.

Mechanisms leading to mitochondrial dysfunction in circulating lymphocytes remain unclear. In the vein of muscular mitochondrial dysfunction, the influence of septic plasma has been suggested. Indeed, Belikova et al. observed a lower response to ADP stimulation and an increased fraction of decoupling oxygen consumption in septic PBMC and in healthy PBMC after incubation in septic plasma, whereas septic PBMC incubated in healthy plasma partially recovered normal respiratory capacities [81]. Plasma factors involved in mitochondrial dysfunction may include $\mathrm{NO}$, ROS [82], and mitochondrial fractions themselves (mtDNA, formyl peptide, and Tfam). Indeed, as previously described, increased mitochondrial ROS production induces mtDNA fragmentation and the release of fragmented mtDNA in both the cytoplasm and the extracellular space. Acting as a DAMP, mtDNA then binds TLR9 on neutrophil plasma membrane and induces both remote organ injury [83] and immunosuppression [84].

Functional alterations in PBMCs may also account for septic shock-induced immunosuppression. For instance, Weiss et al. demonstrated that mitochondrial SRC was reduced and uncoupled respiration was increased in septic shock children PBMCs compared to controls, suggesting that mitochondria within immune cells could not keep pace with an increase in energy demand [85]. If SRC is not sufficient to meet the sepsis-induced increase in metabolic demand, PBMCs may experience an energetic exhaustion, leading to immunoparalysis. Thus, mitochondrial energy failure may be, at least in part, responsible for the inability of the septic PBMCs to respond adequately to pathogen invasion.

3.3.3. Functional Aspects of Lymphocyte Mitochondrial Biogenesis after Sepsis. In septic shock survivors, mitochondrial recovery, and biogenesis have been suggested [24] (Figure 3). After an initial drop, Sjövall et al. observed subsequent increase in cell respiratory capacity in septic patients after admission in intensive care unit (ICU). As early as $48 \mathrm{~h}$ after ICU admission, baseline oxygen consumption had increased by $27 \%$ in septic compared to healthy PBMC [86]. At day 7 , baseline oxygen consumption in septic cells had risen by $73 \%$ compared with control. These modifications were associated with enhanced mitochondrial biogenesis biomarkers: citrate synthase activity, mtDNA, and cytochrome c [86]. As a result, mitochondrial respiration, when expressed in relation to citrate synthase activity, displayed a $27 \%$ to $52 \%$ reduction. Increased oxygen consumption rather reflected an increased mitochondrial content than an empowered ETC. 
Moreover, increase of mitochondrial biogenesis was associated with lymphocytes functional improvements, such as CD8+ $\mathrm{T}$ cell memory development in mice [87]. CD8+ T memory cells had a higher SRC (the extra capacity available in cells to produce energy in response to increased stress), correlated with mitochondrial biogenesis. Similar findings were found in paediatric septic shock patients by Weiss et al. [85].

These results suggest that initial mitochondrial dysfunction in sepsis would induce both quantitative and qualitative impairments of immune cells and that mitochondrial biogenesis may subsequently compensate for these deficiencies by an increased mitochondrial mass and a higher resilience to stressors.

\subsection{Synergic Deleterious Effects of Sepsis-Induced, Mitochon-} drial-Driven Immunosuppression and Acquired Muscle Weakness. Even if a causative link still has to be demonstrated between sepsis-induced muscle weakness/immunoparalysis on the one hand and increased incidence of ventilatoracquired pneumonia on the other hand, many indices converge to a common pathophysiologic pathway. Indeed, sepsisinduced ICU-acquired muscle weakness triggers diaphragmatic dysfunction, weaning failure, and prolonged mechanical ventilation, which is associated with increased mortality when weaning is prolonged above 7 days [88]. In addition, patients suffering from ICU-acquired muscle paresis have a twice larger risk of recurrent respiratory failure [55]. ICUacquired muscle weakness also results in significant atrophy of the pharyngeal, laryngeal, and accessory inspiratory muscles and triggers ICU-acquired swallowing disorders, reduced cough strength, and limited glottis clearance [89]. As dysphagia predisposes to microaspirations in extubated ICU patients, swallowing disorders are clearly associated with a composite outcome of pneumonia, reintubation, or death (adjusted odds ratio, 3.31 [1.78-4.56]; $P<0.01$ ) [90].

Actually, swallowing disorders and reduced cough strength decrease airway clearance and increase the pathogen load (bacterial, viral, and fungal) to the lungs. If immune response effectors are also worn-out, pathogens can develop unrestrainedly. From the immunological point of view, severe persistent sepsis-induced lymphopenia was associated with increased development of secondary infections and death [91]. Sepsis-induced, mitochondrial-driven lymphocyte apoptosis triggers an increased uptake of apoptotic cells by surviving monocytes, macrophages, and dendritic cells, which cannot fight the remaining pathogenic organisms [92]. In this way, sepsis-induced mitochondrial-driven muscle dysfunction and sepsis-induced mitochondrial-driven immunosuppression fuel a feed-forward cycle, culminating in the development of ICU-acquired, healthcare-associated infections, particularly ventilator-associated pneumonias.

\section{Mitochondrial-Targeted Therapies to Promote Mitohormesis in Skeletal Muscles and PBMC after Septic Shock}

As mitochondrial dysfunction in skeletal muscle and PBMC potentially contributes to protracted weaning from mechanical ventilation and persistent infectious foci in patients recovering from septic shock, therapies promoting mitochondrial healing may favour recovery of both muscular functions and immunologic potency. Efforts should be made to focus on the good treatment in the right place and at the appropriate time.

4.1. Early Hemodynamic Resuscitation. Even if restricted oxygen uptake and global tissue hypoxia may trigger inflammatory cascade at the onset of sepsis [93] and if early goaldirected therapy (EGDT) improves biomarkers patterns [94] and patient outcome [9] in the early stages, the "window of opportunity" for EGDT to promote mitochondrial hormesis appears to be narrow and not reproducible in every setting [95]. Indeed, many studies report increased oxygen availability in resuscitated septic shock, in both experimental [96] and clinical [97] settings. Corrêa et al. observed that treatment delay beyond six hours after the onset of septic shock reduced skeletal muscle ATP content in swine [98]. Norepinephrine treatment in endotoxaemic pigs did not increase hepatosplanchnic flow, oxygen delivery, or consumption and did not improve the hepatic lactate extraction ratio. However, norepinephrine increased the liver mitochondria complex I-dependent and complex II-dependent respiratory control ratios [99]. The question as to whether EGDT could reduce mitochondrial dysfunction in skeletal muscles and PBMCs in septic shock patients still remains unresolved.

4.2. Substrate and Cofactor Provision. As complex I is predominantly damaged during sepsis [44], Protti et al. hypothesized that adding succinate, the specific complex II substrate would increase electron flow through the distal part of the ETC and improve mitochondrial oxygen consumption. In soleus muscles harvested from septic rats, the authors demonstrated that succinate provision bypassed the predominant sepsis-induced ETC inhibition (occurring at complex I) and restored oxygen consumption and ATP synthesis to nonseptic levels [100]. To our knowledge, this interesting pathway has not been yet translated to therapeutic strategies in septic shock patients.

The process of $\beta$-oxidation involves the breakdown of fatty acids to generate acetyl-CoA, NADH, and $\mathrm{FADH}_{2}$ and requires $\mathrm{L}$-carnitine for the transport of long-chain cytosolic fatty acids into mitochondria for $\beta$-oxidation [101]. Van der Windt et al. suggest that drugs that target mitochondrial respiratory capacity in PBMCs (such as carnitine palmitoyl transferase) could hold promise as immunotherapeutics and might warrant further study for their ability to alter $\mathrm{T}$ cell responses.

4.3. Mitochondrial Antioxidants and ROS Scavengers. Resveratrol, a natural phenol within red grapes [102], exerts beneficial effects in experimental sepsis when administered either before or shortly after the septic insult [103]. Recent research has demonstrated that resveratrol activates silent mating type information regulator 2 homolog 1 (SIRT1) in mice, which is a key regulator of cellular defences and mitochondrial hormesis in response to metabolic stress [104]. Resveratrol has also been shown to downregulate the proinflammatory response and to have antioxidant properties in aging rats [103]. 
Mitochondrial biogenesis restores oxidative metabolism and thus enhances prosurvival during Staphylococcus aureus sepsis in mice [71].

However, some antioxidants may fail to induce mitohormesis, due to inadequate mitochondrial upload, and mitochondrial-targeted therapies hold promises. In mechanically ventilated mice, Picard et al. demonstrated that transgenic overexpression of a mitochondria-localized antioxidant (peroxiredoxin-3, downregulated in mechanically ventilated human diaphragms) was protective against ventilationinduced diaphragmatic dysfunction [60]. In skeletal muscle, melatonin (a powerful antioxidant) might counteract inducible mtNOS-triggered dysfunction of the ETC, as recently demonstrated in septic mice [105]. Other mitochondria-targeted lipophilic antioxidants like coenzyme $\mathrm{Q}_{10}$ improved mitochondrial biogenesis in LPS-treated mice by activating PGC- $1 \alpha$ and Tfam [106]. One such approach covalently links biomolecules to lipophilic triphenylphosphonium cation $(\mathrm{TPP}+)$. Due to a positive charge, the molecules are driven by the mitochondrial membrane potential to accumulate solely in mitochondria [107]. In their murine septic shock experiment, Zang et al. tested a single, postinfection administration of mitochondria-targeted antioxidant (Mito-Vit-E). Interestingly, Mito-Vit-E provided mitochondria-specific antioxidant defense and improved mitochondrial respiratory function in the heart over time with sepsis, while cytosolic-targeted Vit-E had no such effect [107]. To date, no clinical study supports these encouraging preclinical findings.

Among research possibilities to confirm the key role of initial mitochondrial dysfunction in sepsis, antioxidants could therefore be used to improve mitochondrial work at an early stage of sepsis, in order to diminish mitochondrialmediated ROS production and apoptosis. Besides, regulation of genes involved in lymphocytes mitochondrial-mediated apoptosis (e.g., Bcl-2) and TLR2-mitochondria axis deserve to be more studied [108].

\section{Conclusion}

The "tissue hypoxia" paradigm in sepsis has recently been challenged and should prompt research in the field of cytopathic hypoxia. In septic shock patients, a circulating factor (which could be nitric oxide, superoxide anion, peroxynitrite, or mtDNA) induces mitochondrial ETC dysfunction and increases apoptosis in both myocytes and PBMCs. These processes profoundly impair organ recovery and patient rehabilitation. Therapeutic induction of mitochondrial hormesis (increased mitochondrial mass and a higher resilience to stressors) may be a way to overcome the septic episode and might stand for a promising research to promote organ recovery in critical illness.

\section{List of Abbreviations}

Akt: Protein kinase B

ATP: Adenosine triphosphate

CARS: Compensatory anti-inflammatory response syndrome
$\Delta \Psi_{\mathrm{m}}: \quad$ Mitochondrial transmembrane electron potential

ETC: Electron transport chain

EGDT: Early goal-directed therapy

$\mathrm{FAD}^{+}$: Oxidized flavin-adenine dinucleotide

$\mathrm{FADH}_{2}$ : Reduced flavin-adenine dinucleotide

FCCP: Carbonyl cyanide-4-(trifluoromethoxy)phenylhydrazone

GSK-3 $\beta$ : Glycogen synthase kinase $3 \beta$

ICU: Intensive care unit

iNOS: Inducible nitric oxide synthase

MOF: Multiorgan failure

mPTP: Mitochondrial permeability transition pore

mtDNA: Mitochondrial desoxyribonucleic acid

$\mathrm{NAD}^{+}$: Oxidized nicotinamide adenine dinucleotide

$\mathrm{NADH}$ : Reduced nicotinamide adenine dinucleotide

NO: $\quad$ Nitric oxide

NLRP3: NOD-like receptor family, pyrin domain containing 3

NRF: Nuclear respiratory factors

PBMCs: Peripheral blood mononuclear cells

PGC-1 $\alpha$ : Peroxisome proliferator-activated receptor- $\gamma$ coactivator $1 \alpha$

RNS: $\quad$ Reactive nitrogen species

ROS: Reactive oxygen species

SIRT: Sirtuin

SIRT1: Silent mating type information regulator 2 homolog 1

$\mathrm{SOD}_{2}$ : Manganese superoxide dismutase

SOFA: Sepsis-Related Organ Failure Assessment

SRC: $\quad$ Spare respiratory capacity

Tfam: Mitochondrial transcription factor A

TLR: Toll-like receptor.

\section{Conflicts of Interest}

The authors declare that there are no conflicts of interest regarding the publication of this paper.

\section{Authors' Contributions}

Quentin Maestraggi and Benjamin Lebas were involved in the writing of the first section of the review. Raphaël ClereJehl, Thiên-Nga Chamaraux-Tran, and Pierre-Olivier Ludes were involved in the writing of the second section of the review. Francis Schneider, Pierre Diemunsch, and Bernard Geny were involved in drafting the manuscript and revising it critically for important intellectual content. Julien Pottecher conceived the review and drafted the manuscript. All authors read and approved the final manuscript.

\section{References}

[1] M. Singer, C. S. Deutschman, C. W. Seymour et al., "The third international consensus definitions for sepsis and septic shock (sepsis-3)," The Journal of the American Medical Association, vol. 315, no. 8, pp. 801-810, 2016.

[2] R. C. Bone, R. A. Balk, F. B. Cerra et al., "Definitions for sepsis and organ failure and guidelines for the use of innovative 
therapies in sepsis. The ACCP/SCCM Consensus Conference Committee. American College of Chest Physicians/Society of Critical Care Medicine," Chest, vol. 101, no. 6, pp. 1644-1655, 1992.

[3] M. M. Levy, M. P. Fink, J. C. Marshall et al., “2001 SCCM/ESICM/ACCP/ATS/SIS international sepsis definitions conference," Critical Care Medicine, vol. 31, no. 4, pp. 1250-1256, 2003.

[4] M. Shankar-Hari, G. S. Phillips, M. L. Levy et al., "Developing a new definition and assessing new clinical criteria for septic shock," The Journal of the American Medical Association, vol. 315, no. 8, pp. 775-787, 2016.

[5] J.-L. Vincent, R. Moreno, J. Takala et al., "The SOFA (Sepsisrelated Organ Failure Assessment) score to describe organ dysfunction/failure," Intensive Care Medicine, vol. 22, no. 7, pp. 707-710, 1996.

[6] R. J. Levy, "Mitochondrial dysfunction, bioenergetic impairment, and metabolic down-regulation in sepsis," Shock, vol. 28, no. 1, pp. 24-28, 2007.

[7] E. Abraham and M. Singer, "Mechanisms of sepsis-induced organ dysfunction," Critical Care Medicine, vol. 35, no. 10, pp. 2408-2416, 2007.

[8] R. S. Hotchkiss and I. E. Karl, "The pathophysiology and treatment of sepsis," The New England Journal of Medicine, vol. 348, no. 2, pp. 138-150, 2003.

[9] E. Rivers, B. Nguyen, S. Havstad et al., "Early goal-directed therapy in the treatment of severe sepsis and septic shock," The New England Journal of Medicine, vol. 345, no. 19, pp. 1368-1377, 2001.

[10] D. De Backer, D. O. Cortes, K. Donadello, and J.-L. Vincent, "Pathophysiology of microcirculatory dysfunction and the pathogenesis of septic shock," Virulence, vol. 5, no. 1, pp. 73-79, 2014.

[11] M. P. Fink, "Bench-to-bedside review: cytopathic hypoxia," Critical Care, vol. 6, no. 6, pp. 491-499, 2002.

[12] I. Lee and M. Hüttemann, "Energy crisis: the role of oxidative phosphorylation in acute inflammation and sepsis," Biochimica et Biophysica Acta-Molecular Basis of Disease, vol. 1842, no. 9, pp. 1579-1586, 2014.

[13] D. Brealey, S. Karyampudi, T. S. Jacques et al., "Mitochondrial dysfunction in a long-term rodent model of sepsis and organ failure," American Journal of Physiology-Regulatory Integrative and Comparative Physiology, vol. 286, no. 3, pp. R491-R497, 2004.

[14] B. B. Peruchi, F. Petronilho, H. A. Rojas et al., "Skeletal muscle electron transport chain dysfunction after sepsis in rats," Journal of Surgical Research, vol. 167, no. 2, pp. e333-e338, 2011.

[15] L. A. Callahan and G. S. Supinski, "Sepsis-induced myopathy," Critical Care Medicine, vol. 37, no. 10, pp. S354-S367, 2009.

[16] G. Garrabou, C. Morén, S. López et al., “The effects of sepsis on mitochondria," Journal of Infectious Diseases, vol. 205, no. 3, pp. 392-400, 2012.

[17] M. Hüttemann, S. Helling, T. H. Sanderson et al., "Regulation of mitochondrial respiration and apoptosis through cell signaling: cytochrome $\mathrm{c}$ oxidase and cytochrome $\mathrm{c}$ in ischemia/reperfusion injury and inflammation," Biochimica et Biophysica Acta, vol. 1817, no. 4, pp. 598-609, 2012.

[18] C. Richter, V. Gogvadze, R. Laffranchi et al., "Oxidants in mitochondria: from physiology to diseases," Biochimica et Biophysica Acta-Molecular Basis of Disease, vol. 1271, no. 1, pp. 67-74, 1995.
[19] A. Lejay, F. Fang, R. John et al., "Ischemia reperfusion injury, ischemic conditioning and diabetes mellitus," Journal of Molecular and Cellular Cardiology, vol. 91, pp. 11-22, 2016.

[20] H. F. Galley, "Oxidative stress and mitochondrial dysfunction in sepsis," British Journal of Anaesthesia, vol. 107, no. 1, pp. 57-64, 2011.

[21] J. M. Matés, C. Pérez-Gómez, and I. N. de Castro, "Antioxidant enzymes and human diseases," Clinical Biochemistry, vol. 32, no. 8, pp. 595-603, 1999.

[22] I. Manoli, S. Alesci, M. R. Blackman, Y. A. Su, O. M. Rennert, and G. P. Chrousos, "Mitochondria as key components of the stress response," Trends in Endocrinology and Metabolism, vol. 18, no. 5, pp. 190-198, 2007.

[23] T. F. Liu, C. M. Brown, M. E. Gazzar et al., "Fueling the flame: bioenergy couples metabolism and inflammation," Journal of Leukocyte Biology, vol. 92, no. 3, pp. 499-507, 2012.

[24] C. A. Piantadosi and H. B. Suliman, "Transcriptional control of mitochondrial biogenesis and its interface with inflammatory processes," Biochimica et Biophysica Acta, vol. 1820, no. 4, pp. 532-541, 2012.

[25] M. R. Duchen, "Mitochondria in health and disease: perspectives on a new mitochondrial biology," Molecular Aspects of Medicine, vol. 25, no. 4, pp. 365-451, 2004.

[26] S. Javadov and M. Karmazyn, "Mitochondrial permeability transition pore opening as an endpoint to initiate cell death and as a putative target for cardioprotection," Cellular Physiology and Biochemistry, vol. 20, no. 1-4, pp. 1-22, 2007.

[27] A. Lejay, A. Meyer, A.-I. Schlagowski et al., "Mitochondria: mitochondrial participation in ischemia-reperfusion injury in skeletal muscle," The International Journal of Biochemistry \& Cell Biology, vol. 50, pp. 101-105, 2014.

[28] C. F. Wenceslau, C. G. McCarthy, T. Szasz, K. Spitler, S. Goulopoulou, and R. C. Webb, "Mitochondrial damageassociated molecular patterns and vascular function," European Heart Journal, vol. 35, no. 18, pp. 1172-1177, 2014.

[29] C. Quoilin, A. Mouithys-Mickalad, S. Lécart, M.-P. FontaineAupart, and M. Hoebeke, "Evidence of oxidative stress and mitochondrial respiratory chain dysfunction in an in vitro model of sepsis-induced kidney injury," Biochimica et Biophysica Acta, vol. 1837, no. 10, pp. 1790-1800, 2014.

[30] S. Moncada and J. D. Erusalimsky, "Does nitric oxide modulate mitochondrial energy generation and apoptosis?" Nature Reviews Molecular Cell Biology, vol. 3, no. 3, pp. 214-220, 2002.

[31] B. Beltrán, A. Orsi, E. Clementi, and S. Moncada, "Oxidative stress and S-nitrosylation of proteins in cells," British Journal of Pharmacology, vol. 129, no. 5, pp. 953-960, 2000.

[32] S. W. Ballinger, C. Patterson, C.-N. Yan et al., "Hydrogen peroxide- and peroxynitrite-induced mitochondrial DNA damage and dysfunction in vascular endothelial and smooth muscle cells," Circulation Research, vol. 86, no. 9, pp. 960-966, 2000.

[33] P. T. Schumacker, M. N. Gillespie, K. Nakahira et al., "Mitochondria in lung biology and pathology: more than just a powerhouse," American Journal of Physiology-Lung Cellular and Molecular Physiology, vol. 306, no. 11, pp. L962-L974, 2014.

[34] J. L. Kuck, B. O. Obiako, O. M. Gorodnya et al., "Mitochondrial DNA damage-associated molecular patterns mediate a feedforward cycle of bacteria-induced vascular injury in perfused rat lungs," American Journal of Physiology-Lung Cellular and Molecular Physiology, vol. 308, no. 10, pp. L1078-L1085, 2015.

[35] A. P. West, G. S. Shadel, and S. Ghosh, "Mitochondria in innate immune responses," Nature Reviews Immunology, vol. 11, no. 6, pp. 389-402, 2011. 
[36] C. S. Calfee and M. A. Matthay, "Clinical immunology: culprits with evolutionary ties," Nature, vol. 464, no. 7285, pp. 41-42, 2010.

[37] A. D. Cherry, H. B. Suliman, R. R. Bartz, and C. A. Piantadosi, "Peroxisome proliferator-activated receptor $\gamma$ co-activator 1$\alpha$ as a critical co-activator of the murine hepatic oxidative stress response and mitochondrial biogenesis in Staphylococcus aureus sepsis," Journal of Biological Chemistry, vol. 289, no. 1, pp. 41-52, 2014.

[38] N. K. Patil, N. Parajuli, L. A. MacMillan-Crow, and P. R. Mayeux, "Inactivation of renal mitochondrial respiratory complexes and manganese superoxide dismutase during sepsis: mitochondriatargeted antioxidant mitigates injury," American Journal of Physiology-Renal Physiology, vol. 306, no. 7, pp. F734-F743, 2014.

[39] A. Choumar, A. Tarhuni, P. Lettéron et al., "Lipopolysaccharideinduced mitochondrial DNA depletion," Antioxidants and Redox Signaling, vol. 15, no. 11, pp. 2837-2854, 2011.

[40] R. S. Hotchkiss, A. Strasser, J. E. McDunn, and P. E. Swanson, "Cell death," The New England Journal of Medicine, vol. 361, no. 16, pp. 1570-1583, 2009.

[41] M. Singer, "Mitochondrial function in sepsis: acute phase versus multiple organ failure," Critical Care Medicine, vol. 35, no. 9, pp. S441-S448, 2007.

[42] M. Boulos, M. E. Astiz, R. S. Barua, and M. Osman, "Impaired mitochondrial function induced by serum from septic shock patients is attenuated by inhibition of nitric oxide synthase and poly(ADP-ribose) synthase," Critical Care Medicine, vol. 31, no. 2, pp. 353-358, 2003.

[43] D. Brealey, M. Brand, I. Hargreaves et al., "Association between mitochondrial dysfunction and severity and outcome of septic shock," The Lancet, vol. 360, no. 9328, pp. 219-223, 2002.

[44] C. C. Dahm, K. Moore, and M. P. Murphy, "Persistent Snitrosation of complex I and other mitochondrial membrane proteins by S-nitrosothiols but not nitric oxide or peroxynitrite: Implications for the interaction of nitric oxide with mitochondria," Journal of Biological Chemistry, vol. 281, no. 15, pp. 1005610065, 2006.

[45] G. C. Brown and V. Borutaite, "Inhibition of mitochondrial respiratory complex I by nitric oxide, peroxynitrite and Snitrosothiols," Biochimica et Biophysica Acta-Bioenergetics, vol. 1658, no. 1-2, pp. 44-49, 2004.

[46] E. Nisoli and M. O. Carruba, "Nitric oxide and mitochondrial biogenesis," Journal of Cell Science, vol. 119, no. 14, pp. 28552862, 2006.

[47] R. R. Bartz, P. Fu, H. B. Suliman et al., "Staphylococcus aureus sepsis induces early renal mitochondrial DNA repair and mitochondrial biogenesis in mice," PLoS ONE, vol. 9, no. 7, Article ID e100912, 2014.

[48] J. M. Cuesta and M. Singer, "The stress response and critical illness: a review," Critical Care Medicine, vol. 40, no. 12, pp. 32833289, 2012.

[49] S. V. Baudouin, D. Saunders, W. Tiangyou et al., "Mitochondrial DNA and survival after sepsis: a prospective study," The Lancet, vol. 366, no. 9503, pp. 2118-2121, 2005.

[50] J. E. Carré, J.-C. Orban, L. Re et al., "Survival in critical illness is associated with early activation of mitochondrial biogenesis," American Journal of Respiratory and Critical Care Medicine, vol. 182, no. 6, pp. 745-751, 2010.

[51] O. Friedrich, M. B. Reid, G. Van Den Berghe et al., “The sick and the weak: neuropathies/ myopathies in the critically ill,"
Physiological Reviews, vol. 95, no. 3, article no. A09, pp. 10251109, 2015.

[52] J. Batt, C. C. Dos Santos, J. I. Cameron, and M. S. Herridge, "Intensive care unit-acquired weakness clinical phenotypes and molecular mechanisms," American Journal of Respiratory and Critical Care Medicine, vol. 187, no. 3, pp. 238-246, 2013.

[53] J. Batt, M. Herridge, and C. dos Santos, "Mechanism of ICUacquired weakness: skeletal muscle loss in critical illness," Intensive Care Medicine, vol. 187, no. 10, p. 238, 2017.

[54] A. Demoule, B. Jung, H. Prodanovic et al., "Diaphragm dysfunction on admission to the intensive care unit: prevalence, risk factors, and prognostic impact-a prospective study," American Journal of Respiratory and Critical Care Medicine, vol. 188, no. 2, pp. 213-219, 2013.

[55] N. A. Ali, J. M. O’Brien Jr., S. P. Hoffmann et al., "Acquired weakness, handgrip strength, and mortality in critically III patients," American Journal of Respiratory and Critical Care Medicine, vol. 178, no. 3, pp. 261-268, 2008.

[56] T. J. Iwashyna, E. W. Ely, D. M. Smith, and K. M. Langa, "Longterm cognitive impairment and functional disability among survivors of severe sepsis," JAMA-Journal of the American Medical Association, vol. 304, no. 16, pp. 1787-1794, 2010.

[57] P. S. Zolfaghari, J. E. Carré, N. Parker, N. A. Curtin, M. R. Duchen, and M. Singer, "Skeletal muscle dysfunction is associated with derangements in mitochondrial bioenergetics (but not UCP3) in a rodent model of sepsis," American Journal of Physiology-Endocrinology and Metabolism, vol. 308, no. 9, pp. E713-E725, 2015.

[58] S. K. Powers, A. N. Kavazis, and S. Levine, "Prolonged mechanical ventilation alters diaphragmatic structure and function," Critical Care Medicine, vol. 37, no. 10, pp. S347-S353, 2009.

[59] N. Bernard, S. Matecki, G. Py, S. Lopez, J. Mercier, and X. Capdevila, "Effects of prolonged mechanical ventilation on respiratory muscle ultrastructure and mitochondrial respiration in rabbits," Intensive Care Medicine, vol. 29, no. 1, pp. 111-118, 2003.

[60] M. Picard, B. Jung, F. Liang et al., "Mitochondrial dysfunction and lipid accumulation in the human diaphragm during mechanical ventilation," American Journal of Respiratory and Critical Care Medicine, vol. 186, no. 11, pp. 1140-1149, 2012.

[61] M. Mofarrahi, I. Sigala, Y. Guo et al., "Autophagy and skeletal muscles in sepsis," PLoS ONE, vol. 7, no. 10, Article ID e47265, 2012.

[62] C. E. Baldwin and A. D. Bersten, "Alterations in respiratory and limb muscle strength and size in patients with sepsis who are mechanically ventilated," Physical Therapy, vol. 94, no. 1, pp. 6882, 2014.

[63] A. H. V. Remels, H. R. Gosker, P. Schrauwen et al., “TNF- $\alpha$ impairs regulation of muscle oxidative phenotype: implications for cachexia?" FASEB Journal, vol. 24, no. 12, pp. 5052-5062, 2010.

[64] H. W. H. van Hees, W.-J. M.-J. Schellekens, M. Linkels et al., "Plasma from septic shock patients induces loss of muscle protein," Critical Care, vol. 15, no. 5, article R233, 2011.

[65] B. Lebas, J. Pottecher, A. L. Charles et al., "Skeletal muscle mitochondrial dysfunction after short-term septic shock," Acta Physiologica, vol. 214, pp. 63-63, 2015.

[66] J. Grip, T. Jakobsson, N. Tardif, and O. Rooyackers, “The effect of plasma from septic ICU patients on healthy rat muscle mitochondria," Intensive Care Medicine Experimental, pp. 1-8, 2016. 
[67] K. Jiroutková, A. Krajčová, J. Ziak et al., "Mitochondrial function in skeletal muscle of patients with protracted critical illness and ICU-acquired weakness," Critical Care, vol. 19, no. 1, article 448, 2015.

[68] V. Jeger, S. Djafarzadeh, S. M. Jakob, and J. Takala, "Mitochondrial function in sepsis," European Journal of Clinical Investigation, vol. 43, no. 5, pp. 532-542, 2013.

[69] C. R. Brinkmann, L. Jensen, F. Dagnæs-Hansen et al., "Mitochondria and the lectin pathway of complement," Journal of Biological Chemistry, vol. 288, no. 12, pp. 8016-8027, 2013.

[70] K. Timmermans, M. Kox, G. J. Scheffer, and P. Pickkers, "Plasma nuclear and mitochondrial DNA levels, and markers of inflammation, shock, and organ damage in patients with septic shock," Shock, vol. 45, no. 6, pp. 607-612, 2016.

[71] D. W. Haden, H. B. Suliman, M. S. Carraway et al., "Mitochondrial biogenesis restores oxidative metabolism during Staphylococcus aureus sepsis," American Journal of Respiratory and Critical Care Medicine, vol. 176, no. 8, pp. 768-777, 2007.

[72] R. S. Hotchkiss, K. W. Tinsley, P. E. Swanson et al., "Sepsisinduced apoptosis causes progressive profound depletion of $\mathrm{B}$ and $\mathrm{CD} 4^{+} \mathrm{T}$ lymphocytes in humans," Journal of Immunology, vol. 166, no. 11, pp. 6952-6963, 2001.

[73] D. H. Wyllie, I. C. J. W. Bowler, and T. E. A. Peto, "Relation between lymphopenia and bacteraemia in UK adults with medical emergencies," Journal of Clinical Pathology, vol. 57, no. 9, pp. 950-955, 2004.

[74] D. C. Angus and T. van der Poll, "Severe sepsis and septic shock," The New England Journal of Medicine, vol. 369, no. 9, pp. 840851, 2013.

[75] J. S. Boomer, K. To, K. C. Chang et al., "Immunosuppression in patients who die of sepsis and multiple organ failure," Journal of the American Medical Association, vol. 306, no. 23, pp. 25942605, 2011.

[76] K. C. Chang, J. Unsinger, C. G. Davis et al., "Multiple triggers of cell death in sepsis: death receptor and mitochondrial-mediated apoptosis," FASEB Journal, vol. 21, no. 3, pp. 708-719, 2007.

[77] Z.-G. Liu, S.-Y. Ni, G.-M. Chen et al., "Histones-mediated lymphocyte apoptosis during sepsis is dependent on $\mathrm{p} 38$ phosphorylation and mitochondrial permeability transition," PLoS ONE, vol. 8, no. 10, Article ID e77131, 2013.

[78] S. U. Weber, J.-C. Schewe, L. E. Lehmann et al., "Induction of Bim and Bid gene expression during accelerated apoptosis in severe sepsis," Critical Care, vol. 12, no. 5, article R128, 2008.

[79] P. Bilbault, T. Lavaux, A. Launoy et al., "Influence of drotrecogin alpha (activated) infusion on the variation of $\mathrm{Bax} / \mathrm{Bcl}-2$ and $\mathrm{Bax} / \mathrm{Bcl}$-xl ratios in circulating mononuclear cells: a cohort study in septic shock patients," Critical Care Medicine, vol. 35, no. 1, pp. 69-75, 2007.

[80] A. M. Japiassú, A. P. S. A. Santiago, J. D. C. P. D’Avila et al., "Bioenergetic failure of human peripheral blood monocytes in patients with septic shock is mediated by reduced F1Fo adenosine-5/-triphosphate synthase activity," Critical Care Medicine, vol. 39, no. 5, pp. 1056-1063, 2011.

[81] I. Belikova, A. C. Lukaszewicz, V. Faivre, C. Damoisel, M. Singer, and D. Payen, "Oxygen consumption of human peripheral blood mononuclear cells in severe human sepsis," Critical Care Medicine, vol. 35, no. 12, pp. 2702-2708, 2007.

[82] M. Singer, "The role of mitochondrial dysfunction in sepsisinduced multi-organ failure," Virulence, vol. 5, no. 1, pp. 66-72, 2014.
[83] S. Sun, T. Sursal, Y. Adibnia et al., "Mitochondrial DAMPs Increase Endothelial Permeability through neutrophil dependent and independent pathways," PLOS ONE, vol. 8, no. 3, Article ID e59989, 2013.

[84] K. Timmermans, M. Kox, M. Vaneker et al., "Plasma levels of danger-associated molecular patterns are associated with immune suppression in trauma patients," Intensive Care Medicine, vol. 42, no. 4, pp. 551-561, 2016.

[85] S. L. Weiss, M. A. Selak, F. Tuluc et al., "Mitochondrial dysfunction in peripheral blood mononuclear cells in pediatric septic shock," Pediatric Critical Care Medicine, vol. 16, no. 1, pp. e4-e12, 2015.

[86] F. Sjövall, S. Morota, J. Persson, M. J. Hansson, and E. Elmér, "Patients with sepsis exhibit increased mitochondrial respiratory capacity in peripheral blood immune cells," Critical Care, vol. 17, no. 4, article R152, 2013.

[87] G. J. W. van der Windt, B. Everts, C.-H. Chang et al., "Mitochondrial respiratory capacity is a critical regulator of CD8+ T cell memory development," Immunity, vol. 36, no. 1, pp. 68-78, 2012.

[88] O. Peñuelas, F. Frutos-Vivar, C. Fernández et al., "Characteristics and outcomes of ventilated patients according to time to liberation from mechanical ventilation," American Journal of Respiratory and Critical Care Medicine, vol. 184, no. 4, pp. 430437, 2011.

[89] M. Macht, T. Wimbish, C. Bodine, and M. Moss, "ICU-acquired swallowing disorders," Critical Care Medicine, vol. 41, no. 10, pp. 2396-2405, 2013.

[90] M. Macht, T. Wimbish, B. J. Clark et al., "Post-extubation dysphagia is persistent and associated with poor outcomes in survivors of critical illness," Critical Care, vol. 15, no. 5, p. R231, 2011.

[91] A. Drewry, N. Samra, L. Skrupky, B. Fuller, S. Compton, and R. Hotchkiss, "Persistent lymphopenia after diagnosis of sepsis predicts mortality," Shock, vol. 42, no. 5, pp. 383-391, 2014.

[92] R. S. Hotchkiss, G. Monneret, and D. Payen, "Sepsis-induced immunosuppression: from cellular dysfunctions to immunotherapy," Nature Reviews Immunology, vol. 13, no. 12, pp. 862874, 2013.

[93] D. Annane, E. Bellissant, and J. Cavaillon, "Septic shock," The Lancet, vol. 365, no. 9453, pp. 63-78, 2005.

[94] E. P. Rivers, J. A. Kruse, G. Jacobsen et al., “The influence of early hemodynamic optimization on biomarker patterns of severe sepsis and septic shock," Critical Care Medicine, vol. 35, no. 9, pp. 2016-2024, 2007.

[95] ProCESS, D. M. Yealy, J. A. Kellum et al., "A randomized trial of protocol-based care for early septic shock," The New England Journal of Medicine, vol. 370, no. 18, pp. 1683-1693, 2014.

[96] D. M. Rosser, R. P. Stidwill, D. Jacobson, and M. Singer, "Oxygen tension in the bladder epithelium rises in both high and low cardiac output endotoxemic sepsis," Journal of Applied Physiology, vol. 79, no. 6, pp. 1878-1882, 1995.

[97] P. Boekstegers, S. Weidenhofer, T. Kapsner, and K. Werdan, "Skeletal muscle partial pressure of oxygen in patients with sepsis," Critical Care Medicine, vol. 22, no. 4, pp. 640-650, 1994.

[98] T. D. Corrêa, M. Vuda, A. R. Blaser et al., "Effect of treatment delay on disease severity and need for resuscitation in porcine fecal peritonitis," Critical Care Medicine, vol. 40, no. 10, pp. 2841-2849, 2012.

[99] T. Regueira, B. Bänziger, S. Djafarzadeh et al., "Norepinephrine to increase blood pressure in endotoxaemic pigs is associated with improved hepatic mitochondrial respiration," Critical Care, vol. 12, no. 4, article R88, 2008. 
[100] A. Protti, J. Carré, M. T. Frost et al., "Succinate recovers mitochondrial oxygen consumption in septic rat skeletal muscle," Critical Care Medicine, vol. 35, no. 9, pp. 2150-2155, 2007.

[101] A. J. Dare, A. R. J. Phillips, A. J. R. Hickey et al., "A systematic review of experimental treatments for mitochondrial dysfunction in sepsis and multiple organ dysfunction syndrome," Free Radical Biology and Medicine, vol. 47, no. 11, pp. 1517-1525, 2009.

[102] S. Dal-Ros, J. Zoll, A.-L. Lang et al., "Chronic intake of red wine polyphenols by young rats prevents aging-induced endothelial dysfunction and decline in physical performance: role of NADPH oxidase," Biochemical and Biophysical Research Communications, vol. 404, no. 2, pp. 743-749, 2011.

[103] J. Morel and M. Singer, "Statins, fibrates, thiazolidinediones and resveratrol as adjunctive therapies in sepsis: could mitochondria be a common target?" Intensive Care Medicine Experimental, vol. 2, no. 1, pp. 1-9, 2014.

[104] M. Lagouge, C. Argmann, Z. Gerhart-Hines et al., "Resveratrol improves mitochondrial function and protects against metabolicdisease by activating SIRT1 and PGC-1 $\alpha$," Cell, vol. 127, no. 6, pp. 1109-1122, 2006.

[105] G. Escames, L. C. López, V. Tapias et al., "Melatonin counteracts inducible mitochondrial nitric oxide synthase-dependent mitochondrial dysfunction in skeletal muscle of septic mice," Journal of Pineal Research, vol. 40, no. 1, pp. 71-78, 2006.

[106] P. Bullón, L. Román-Malo, F. Marín-Aguilar et al., "Lipophilic antioxidants prevent lipopolysaccharide-induced mitochondrial dysfunction through mitochondrial biogenesis improvement," Pharmacological Research, vol. 91, pp. 1-8, 2015.

[107] Q. S. Zang, H. Sadek, D. L. Maass et al., "Specific inhibition of mitochondrial oxidative stress suppresses inflammation and improves cardiac function in a rat pneumonia-related sepsis model," American Journal of Physiology-Heart and Circulatory Physiology, vol. 302, no. 9, pp. H1847-H1859, 2012.

[108] M. Leone and R. Moreau, "Leukocyte toll-Like receptor 2mitochondria axis in sepsis: unraveling immune response sophistication," Anesthesiology, vol. 121, no. 6, pp. 1147-1149, 2014. 


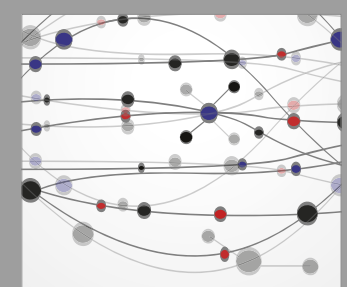

The Scientific World Journal
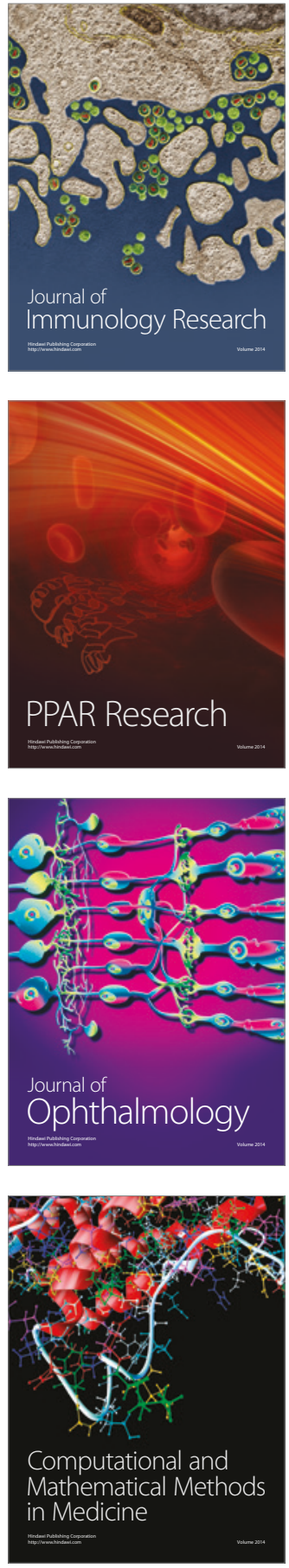

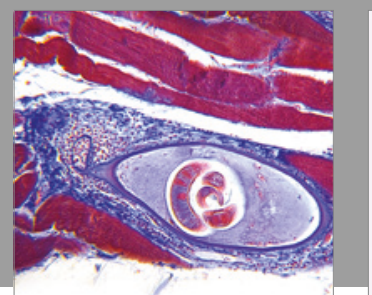

Gastroenterology Research and Practice
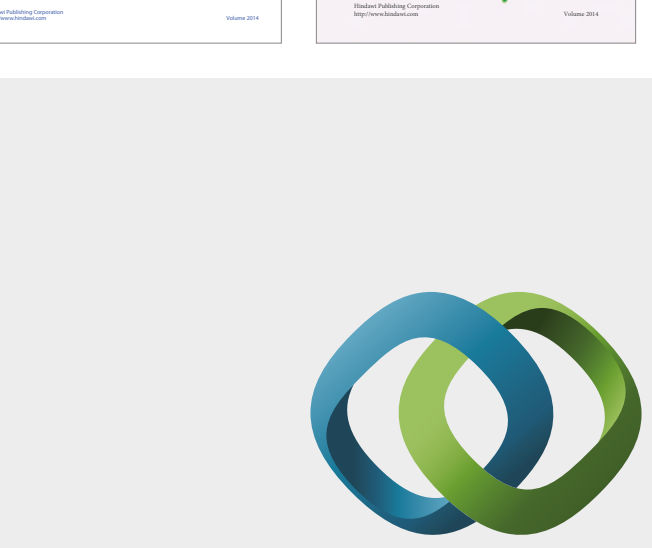

\section{Hindawi}

Submit your manuscripts at

https://www.hindawi.com
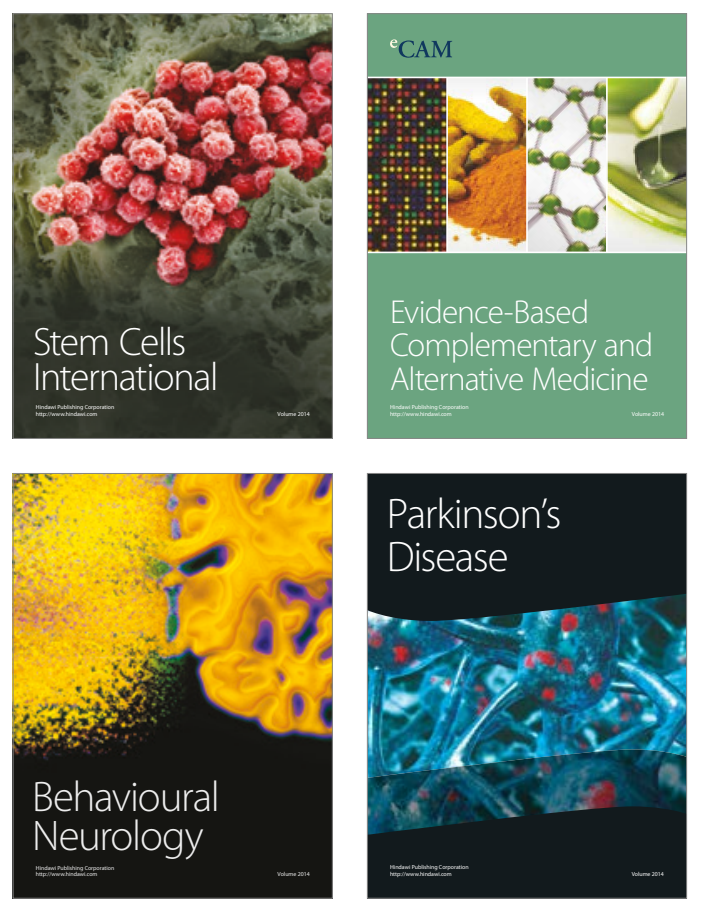
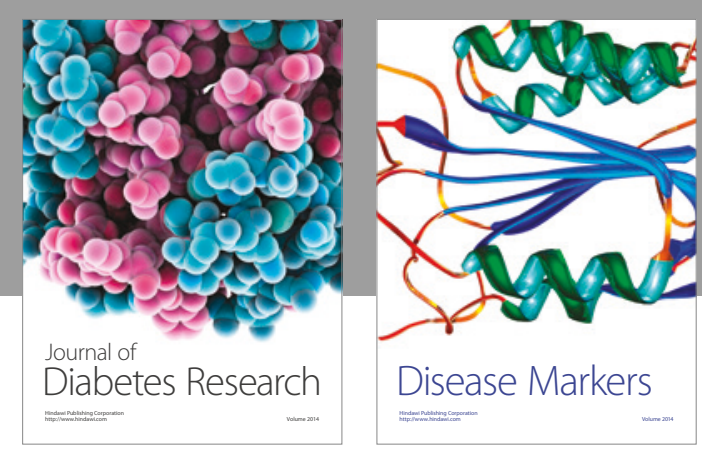

Disease Markers
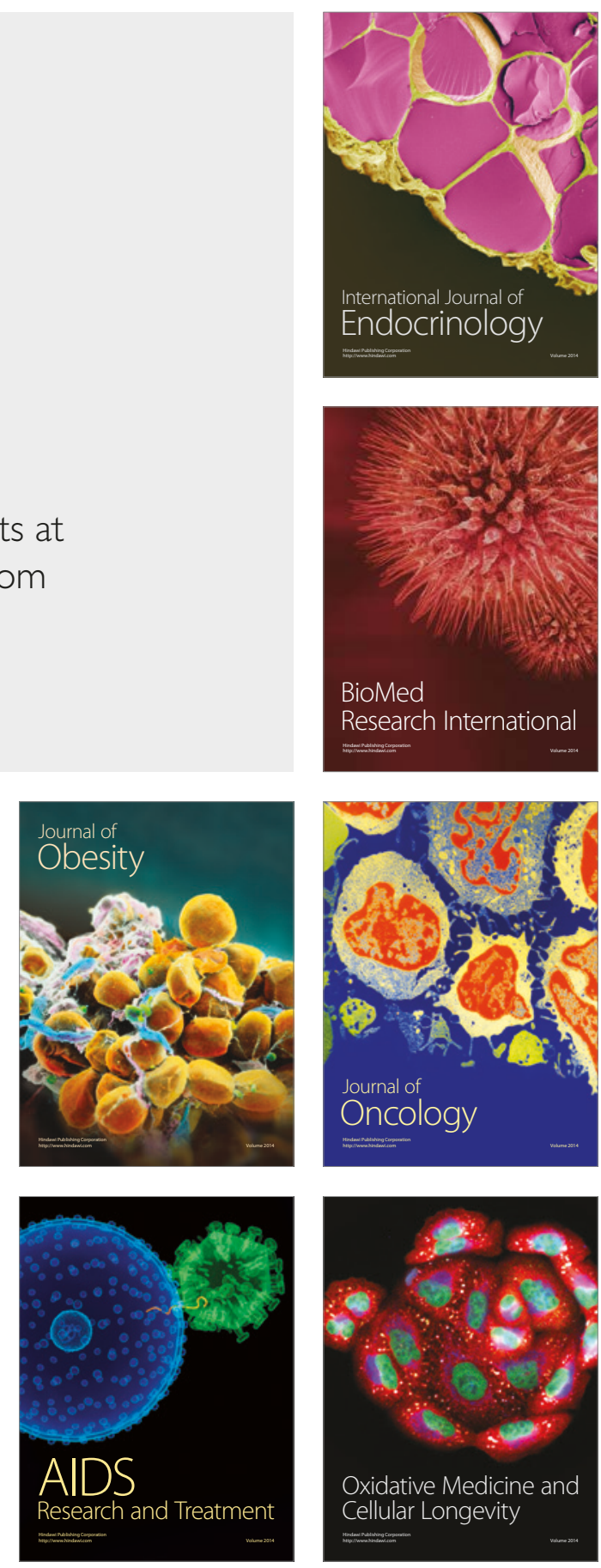